\title{
ENVIRONMENTAL EFFECT OF BUS PRIORITY MEASURES APPLIED ON A ROAD NETWORK IN SANTIAGO, CHILE
}

Xenia Karekla (Corresponding Author)

Department of Civil, Environmental and Geomatic Engineering

Centre of Transport Studies, University College London

Gower Street, London, WC1E 6BT, UK

Email: x.karekla@ucl.ac.uk

Phone: +44 (0) 2076797224

\section{Rodrigo Fernandez}

Faculty of Engineering and Applied Sciences

Universidad de los Andes

Monseñor Álvaro del Portillo 12455, Santiago, Chile

Email: rfa@uandes.cl

18 Phone: +5622618 1143

\section{Nick Tyler}

Department of Civil, Environmental and Geomatic Engineering

Centre of Transport Studies, University College London

23 Gower Street, London, WC1E 6BT, UK

24 Email: n.tyler@ucl.ac.uk

25 Phone: +44 2076791562 
1 ABSTRACT

2 Air pollution is at the highest levels ever and there is currently a worldwide initiative by 3 transport engineers and urban planners in redesigning public transport modes and cities to

4 become more sustainable and environmentally friendly. The environmental impact of everyday

5 activities is more apparent in developing cities which take longer to adapt to advanced methods

6 of running public transport modes. This study aims to investigate the reduction of bus energy

7 consumption and carbon emissions through bus priority measures in a bus route in the city of

8 Santiago, Chile. Two bus priority schemes are tested in this study: Bus Only Lanes and Bus

9 Signal Priority. The microscopic traffic simulator TSIS-CORSIM is used to quantify the

10 environmental impact of these schemes. The results have shown that both schemes lead to lower

11 fuel consumption and emissions, especially for the bus service. The environmental

12 improvements are mostly apparent at traffic flows below $1000 \mathrm{veh} / \mathrm{h}$, with clear benefits for both

13 the bus service and passenger cars when dedicated bus lanes are included in the road

14 infrastructure.

15

16

Keywords: energy consumption; carbon emissions; bus priority; bus lane; bus signal priority;

20 traffic simulation 


\section{INTRODUCTION}

Air pollution is an environmental phenomenon which puts a burden on people's lives and is affecting their everyday activities. Especially in urban metropolises, where more than half of the global population is accommodated (1), 70\% of the world's carbon emissions is recorded (2). The world's $10 \%$ richest people alone emit 50\% of the worldwide greenhouse gas (GHG) emissions (3). These statistics show that the activities undertaken in cities are harming the environment and there is a clear need for changing our habits in the way we attempt everyday activities. It is essential to find ways to reduce air pollution and climate change whilst preserving people's health and improving their wellbeing.

One of the greatest GHG emitters in urban environments, with documented effects on air pollution, is transport systems $(4,5)$. Transporting people and goods is a vital activity in cities, however energy related emissions from transportation amounts to $25 \%$ of GHG emissions worldwide (6). For reference, in 2015 the transportation sector in Europe emitted $26 \%$ of the world's GHG (7) and the USA emitted 27\% (8). Transportation related emissions in China doubled between 2000 and 2010 and an extra 54\% increase is expected until 2020 (9). Road transportation, in particular, is considered responsible for $70 \%$ of all GHG emissions, making it the largest polluter of all transport modes (10). As an example of the Latin American context, in Chile, $28 \%$ of its GHG emissions are generated by the transport sector, and with private cars gaining ground over public transportation year after year, the levels of GHG emissions are constantly increasing. Indicative, $45 \%$ of GHG emissions from transportation in Chile are produced by cars and taxis, $10 \%$ by buses, and $1 \%$ by trains (11).

In transport economics, road pollution is considered as an abuse of the good road users were given whilst other users on the street are passive victims (12). Hence, it is necessary to provide alternative means of transport, such as public transport modes, as well as to improve the existing ones so that people stop the abuse of roads and start favouring environmentally friendly means over their private cars. Enabling people to choose more sustainable means of transport for their everyday mobility could significantly contribute to the reduction of GHG emissions worldwide.

In regards to energy consumption, urban areas consume up to $75 \%$ of global energy (13), the majority of which is absorbed by the transportation sector. In 2010, transportation systems around the world consumed 19\% of global energy supplies, $69 \%$ of which was consumed by road transportation (14). This number is expected to grow rapidly, and by 2050 transport energy consumption is expected to reach an $80 \%$ increase. In other words, by $205030 \%$ of the global energy will be channelled towards transportation, with the highest demand arising from developing countries and areas undergoing strong economic and population growth, such as the Latin America and China. Statistics concerning car ownership rates highlight the global need to increase the attractiveness of public transport modes and to invest towards energy efficient transport systems; car ownership in China has been growing by $12 \%$ per year, by $1.6 \%$ per year in the US, whereas only slight increase of vehicle ownership is expected for the European countries that are members of the Organization for Economic Cooperation and Development (OECD-Europe) by 2050 (14).

Bus priority schemes have been widely tested in various environments, such as in Japan, in the UK, and Canada, with the aim to reduce traffic congestion, to improve bus journey times and road safety (including but not limited to 15-17), but their environmental effect is still being explored. In order to tackle the environmental challenges discussed above, this study's main objective is to examine how bus priority schemes may reduce energy consumption and carbon emissions. It focuses on the bus system in Santiago, Chile, and with the use of the microscopic 
traffic simulator TSIS-CORSIM, the current road network and bus system is replicated. Two bus priority schemes - Bus Only Lanes and Bus Signal Priority - are tested during the morning peak period.

The collection of the data, the model parameters and the examined bus priority schemes are described in Methodology. The results obtained for energy consumption and emissions for each of the schemes are presented in the Results for both the cars and the bus system. The results are then being discussed and the traffic flow and bus priority scheme at which the highest environmental improvement is achieved are proposed.

\section{METHODOLOGY}

Bus route $\mathrm{C} 01$ travels across the district of Las Condes, one of the most affluent districts in Santiago, Chile, and presents one of the highest boarding and alighting rates of bus routes in that area. In addition, the highest car volumes of Santiago are concentrated in the area of Las Condes (18), which is reflected in an increase in road congestion and air pollution. Therefore, these characteristics make the area of Las Condes and the road segment served by bus route C01 an excellent candidate for evaluation.

The operator of bus route C01, Redbus Urbano S.A of the Transdev Group, was contacted in order to provide necessary data regarding the bus system. Bus frequency, number of bus stops and the distance between them, passenger demand during boarding and alighting, bus dwell times, vehicle length, capacity and velocity, were collected. Bus route $\mathrm{C} 01$ is in fact a combination of two bus routes - $\mathrm{C} 01$ and a shorter one, $\mathrm{C} 01 \mathrm{c}$, that serves only the middle stations of the general route in order to increase the service frequency during peak hours - and hence it was decided to study 16 bus stops that are served by both services (Figure 1, a). Only the direction away from the city centre (north-east) was considered, with the first bus station being Juan Montalvo (PC278) and the last being San Francisco De Asís (PC351). The length of the studied network is around $6 \mathrm{~km}$.

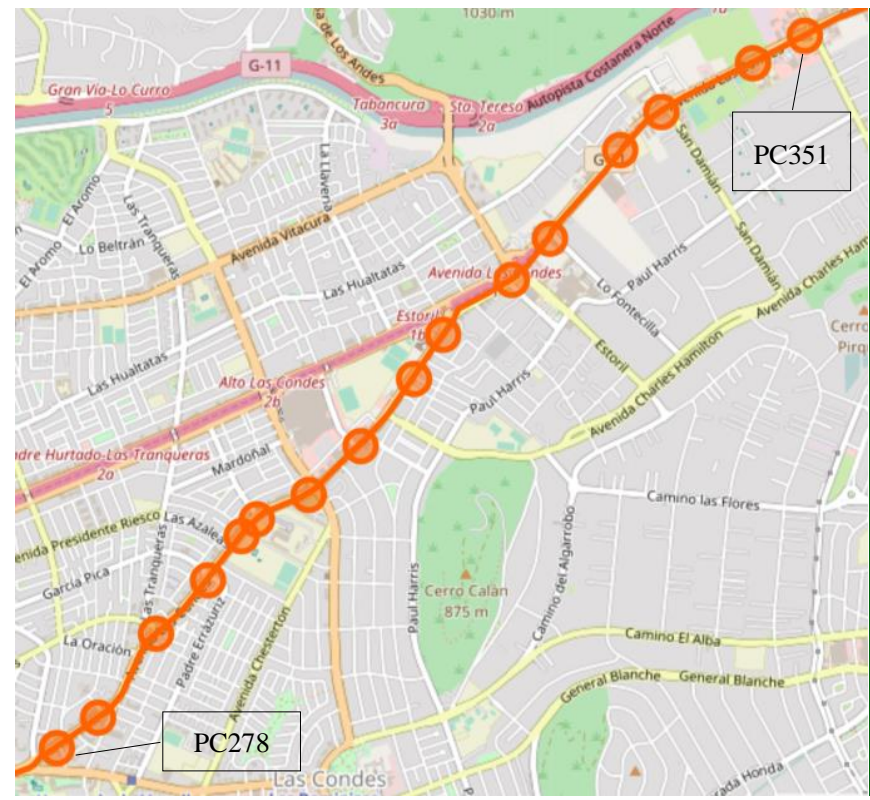

(a)

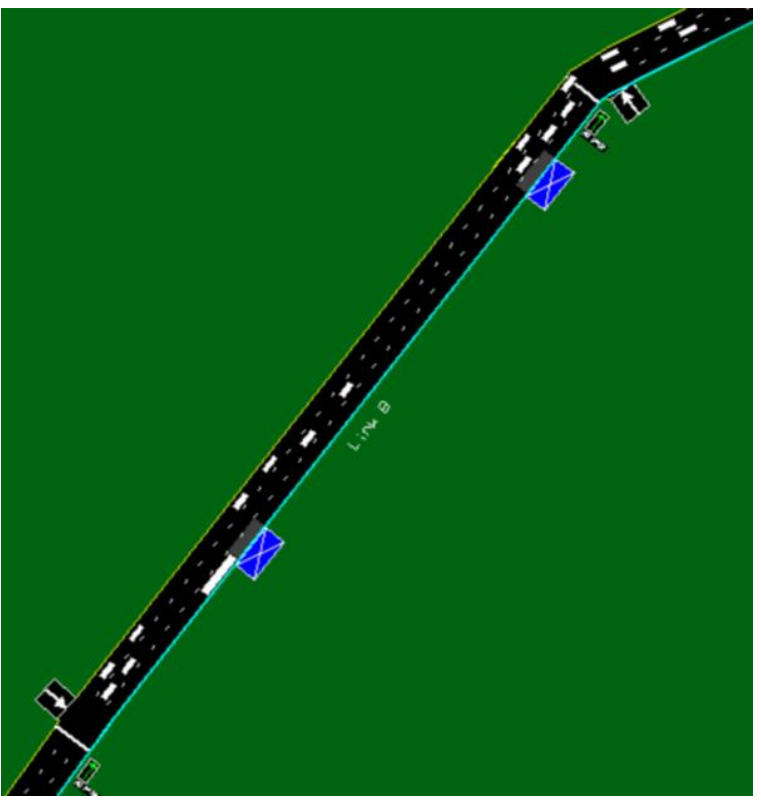

(b) model of part of the network when testing the effect of bus lanes 
On-site observations were also used to gather data regarding traffic flow on the examined road network. Such data involved the number of cars at each section of the network, location of traffic signals (nodes), signal times and cycle times, location of pedestrian crossings and pedestrian flow and road parking. Considering the road infrastructure, data such as road gradient, length of road between traffic signals (links), number of lanes on the street and the coordinates of each node were also collected.

The collected data were organised in a database and were then entered in the simulation package (Figure 1, b). The answer to the research question was attempted through the alteration of three variables: reduction of the current traffic flow and introduction of two bus priority schemes - Bus Only Lanes and Bus Signal Priority (BSP).

1. Traffic flow The current traffic flow along bus route C01 was measured to be $1500 \mathrm{veh} / \mathrm{h}$. Given the frequency of buses on the $\mathrm{C} 01$ bus route ( 26 buses per hour), the maximum flow of cars on this road should not exceed $1000 \mathrm{veh} / \mathrm{h}$ to implement a bus lane (20). Therefore, the environmental effect of lower traffic flows, such as $1000 \mathrm{veh} / \mathrm{h}$ and $800 \mathrm{veh} / \mathrm{h}$ was examined. Although these traffic flows are lower than the current, they do not reflect a free flow condition, but rather a 'medium' level of traffic congestion on the road. Furthermore, a higher traffic flow of 2000 $\mathrm{veh} / \mathrm{h}$ was also tested so as to reveal the trends in a congested environment as well as to have a clearer understanding of the effect of traffic flow on energy consumption and emissions.

\section{Bus Lanes} In regard to road infrastructure, the examined network does not provide dedicated lanes for the bus service and hence a mixed-traffic usage of the road is observed. Having buses travelling on separate lanes to private cars would reveal whether this measure benefits the bus service as well as the environment.

\section{Bus Signal Priority (BSP)} Traffic signals in Avenida Las Condes allow a cycle of 110 sec. To test the particular scheme, traffic signal cycles were reduced to $60 \mathrm{sec}$ to enable buses to spend less time at each traffic signal, and hence reduce their journey time (21). The effect of BSP on energy consumption and emissions was also tested.

The vehicle fleet considered in all three cases consisted of high performance passenger cars (75\% of the examined traffic flow), low performance passenger cars (25\% of the examined traffic flow) and buses. An average occupancy of 1.30 passengers per vehicle was considered for both the high and low performance passenger cars, whereas it was assumed that each bus travelling along the simulated network is carrying 25 passengers on average. The length of each vehicle (high and low passenger car and bus) is $5 \mathrm{~m}, 4.30 \mathrm{~m}$ and $12 \mathrm{~m}$ respectively and the maximum non-emergency acceleration/deceleration of both cars and buses was set at $2.5 \mathrm{~m} / \mathrm{s}^{2}$.

Vehicle energy consumption (L) and emissions (grams/km) are calculated internally based on tabulated data embedded in the TSIS-CORSIM software. Information about the vehicle's performance and speed is used to access the software tables and define the rate of fuel consumption (scaled by 0.0001 gallons/sec, later converted to litres) and emission (scaled by $0.001 \mathrm{grams} / \mathrm{sec}$ ) as a function of the vehicle's acceleration. The methods used for the development of these tables can be found in (22). The effect of each bus priority scheme on vehicles' speed $(\mathrm{km} / \mathrm{h})$ and fuel efficiency $(\mathrm{km} / \mathrm{L})$ was also derived. Enhanced understanding of

45 the way a scheme affects a combination of vehicle performance aspects is important to reach a 
conclusion as to which combination of traffic flow and scheme would be best to be applied in order to improve the environment.

\section{RESULTS}

This section presents the effect of each of the proposed schemes on fuel consumption and emissions, and reference is also made to vehicles' speed and fuel efficiency. The impact of the proposed schemes on GHG emissions specifically is examined through three common diesel pollutants which contribute to global warming and include unburned hydrocarbons (HC), carbon monoxide (CO) and nitrogen oxides (NOx). The results derived from each scheme are discussed in comparison to the current traffic situation at the examined network (mixed-traffic) and the percentage change of each environmental factor between the two schemes is presented. Traffic flow is not presented separately, but in conjunction with each of the bus priority schemes. The numerical results of each case, especially for vehicle speed and fuel efficiency for which graphs are not presented, can be provided upon request.

\section{Scheme 1: Bus Only Lanes}

Having dedicated bus lanes so that buses and cars do not mix on the road has proven beneficial for the environment as well as for its users. First, having buses using a dedicated lane increases the speed of buses up to $11 \%$ compared to the mixed-traffic case. One effect is that the average speed of cars reduces dramatically, especially at high traffic flows $(2000 \mathrm{veh} / \mathrm{h})$ where a reduction of $30 \%$ is observed. At lower traffic flows of 1000 or $800 \mathrm{veh} / \mathrm{h}$, which imply a 'medium' level of traffic congestion, the average speed of buses still increases by $4 \%$ and $6 \%$ respectively, whereas the average speed of cars presents the smallest change (reduction of 3\% and 2\% respectively). Thus, it is observed that the gain in speed for the buses is almost equal to the loss in speed for the cars at the lowest traffic flows.

Focusing on fuel consumption (Figure 2, a), enabling buses to travel in dedicated bus lanes whilst traffic flow remains unchanged $(1500 \mathrm{veh} / \mathrm{h})$ reduces the amount of fuel buses need to travel along the examined road network by $2 \%$. However, cars appear to use $6 \%$ more fuel due to the road congestion (their average speed drops by $7 \%)$. At a higher traffic flow $(2000 \mathrm{veh} / \mathrm{h})$ congestion becomes so high that it is inevitable that cars appear to use $53.6 \%$ less fuel (their average speed drops by $30 \%$ and they are almost idle). On the other hand, at a traffic flow of $2000 \mathrm{veh} / \mathrm{h}$, buses move faster but spend less time accelerating and decelerating, hence a $54.2 \%$ decrease in their fuel consumption is observed. At lower traffic flows (1000 and $800 \mathrm{veh} / \mathrm{h})$, fuel consumption for both the buses and cars reduces by $3 \%$ at the most.

Fuel efficiency follows the opposite trend of fuel consumption. When buses travel on dedicated bus lanes they use less fuel in all traffic flow conditions, and hence their fuel efficiency increases. Disregarding the cases at which congestion is very high (1500 and 2000 $v e h / h$ ) and therefore fuel efficiency presents the highest increase, buses benefit the most in terms of fuel efficiency when traffic flow is at $800 \mathrm{veh} / \mathrm{h}$. Cars, on the other hand, present a decrease in fuel efficiency in all traffic flow cases apart from when $800 \mathrm{veh} / \mathrm{h}$ use the road. Although it is a small increase $(+1 \%)$, it is the only case when cars can travel longer distances with the same amount of fuel. 

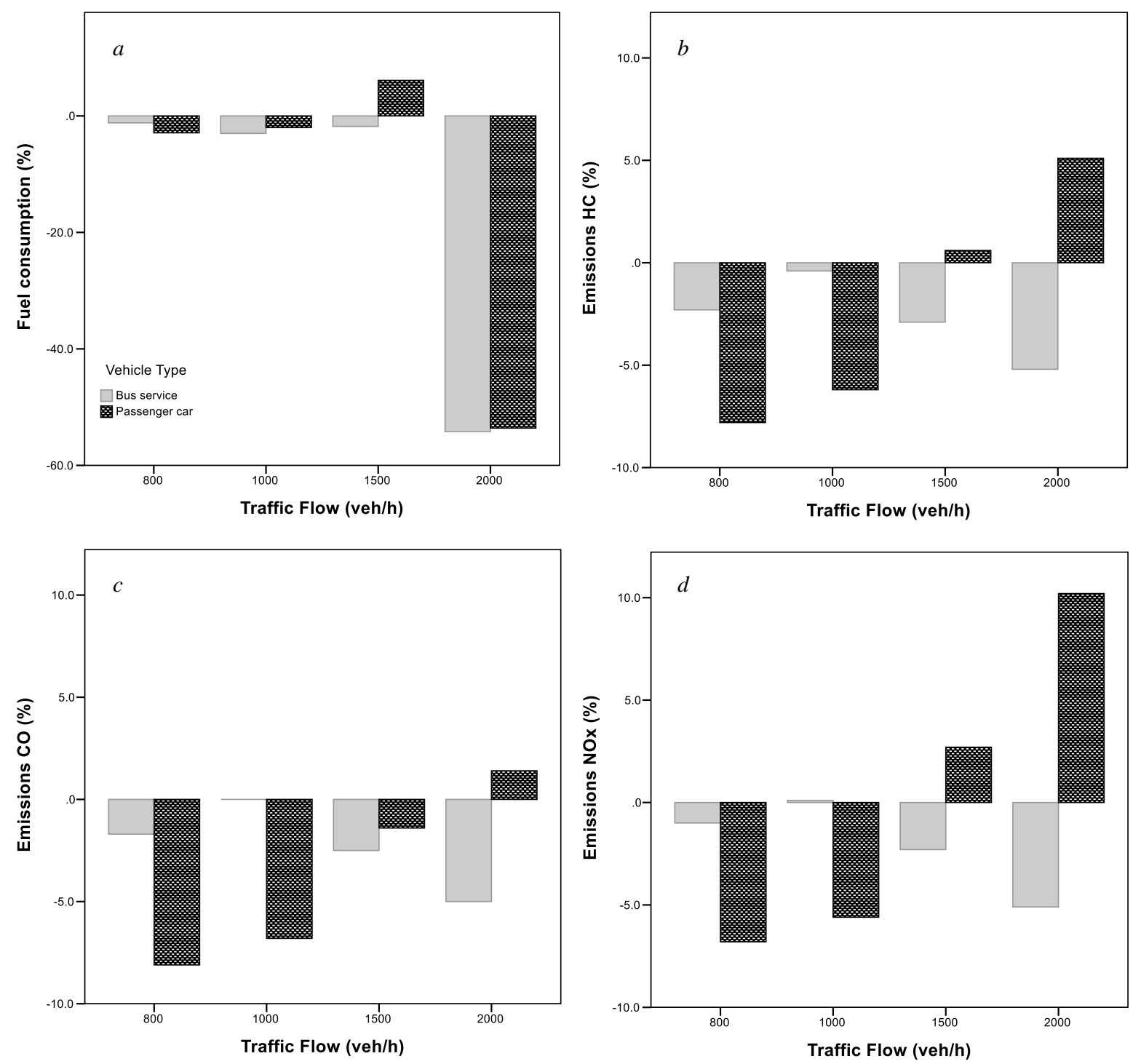

1

FIGURE 2 Percentage change of fuel consumption and emissions for cars and buses when 3 bus lanes are introduced 
1 Regarding pollution (Figure 2, b, c, d), the implementation of a bus lane reduces emissions for both buses and cars, especially at a 'medium' congestion level when traffic flow is less than $1000 \mathrm{veh} / \mathrm{h}$ (the flow for which a bus lane is required). The observed reduction in emissions follows the same tendency for $\mathrm{HC}, \mathrm{CO}$ and NOx gases. The highest decrease in GHG emissions for passenger cars is observed at $800 \mathrm{veh} / \mathrm{h}$ ( $8 \%$ for $\mathrm{HC}$ and $\mathrm{CO}$ and $7 \%$ for NOx). Similarly, the bus service emits the least amount of GHG at $800 \mathrm{veh} / \mathrm{h}$, however the benefit is less than that observed for the cars (decreases of $2 \%$ for $\mathrm{HC}$ and $\mathrm{CO}$ and $1 \%$ for NOx). In the cases of actual flow and high congestion (1500 and $2000 \mathrm{veh} / \mathrm{h}$ ), bus lanes also appear to benefit the environment when buses are taken into account (reduction of $2.5 \%$ for GHG emissions at 1500 $\mathrm{veh} / \mathrm{h}$ and of $5 \%$ at $2000 \mathrm{veh} / \mathrm{h}$ ). However, cars appear to be high emitters of GHG when traffic flow is $1500 \mathrm{veh} / \mathrm{h}$ and higher. Especially at $1500 \mathrm{veh} / \mathrm{h}$, passenger cars emit more $\mathrm{HC}(+0.6 \%)$ and NOx $(+2.7 \%)$ gases, but less $\mathrm{CO}(-1.4 \%)$. This occurs when, during combustion, the quantity of oxygen in the air is higher than normal and creates a lean mixture of air and fuel (air/fuel ratio greater than 1). Due to this, a lower concentration of $\mathrm{CO}$ is produced, and if combustion is not complete and occurs at high temperatures, a higher concentration of $\mathrm{HC}$ and NOx gases is produced (19).

\section{Scheme 2: Bus Signal Priority (BSP)}

In the case of the BSP scheme, the average speed of both cars and buses increases at all examined traffic flows between 6 and $8 \%$ in comparison to the mixed-traffic case. This shows that both buses and cars benefit from this scheme regardless of the congestion on the road. The highest increase of average speed for cars $(8.3 \%)$ is observed at a traffic flow of $1000 \mathrm{veh} / \mathrm{h}$, whereas buses can travel by $7.6 \%$ faster at the current traffic flow $(1500 \mathrm{veh} / \mathrm{h})$ when they are given $\mathrm{BSP}$.

When fuel consumption is put under the microscope (Figure 3, a), both cars and buses benefit from a BSP scheme as less energy is required to travel along the examined network compared to the case when no BSP is given. This effect is seen at all examined traffic flows, however the highest reduction in fuel consumption for cars $(22 \%)$ is observed when $1000 \mathrm{veh} / \mathrm{h}$ are using the road. At this traffic flow, cars can travel up to $4 \%$ longer distances with the same amount of fuel, compared to the current situation $(1500 \mathrm{veh} / \mathrm{h})$. Reduction in fuel consumption for buses is not as significant as it is for cars. At a traffic flow of $1000 \mathrm{veh} /$ hour, where cars present the highest benefit, buses appear to have the lowest reduction of fuel consumption (1.2\%). This might be due to the high average speed they develop $(26.2 \mathrm{~km} / \mathrm{h}$ ) as well as due to the number of stops (bus stops and traffic signals) they make compared to cars. At this level of traffic flow, cars also reach high average speed $(37.3 \mathrm{~km} / \mathrm{h})$ but only stop at the traffic signals. The highest reduction in fuel consumption for buses is observed at $2000 \mathrm{veh} / \mathrm{h}$ and at $800 \mathrm{veh} / \mathrm{h}(1.8 \%)$.

Subsequently, fuel efficiency for cars and buses increases, with the highest change in fuel efficiency for buses observed at the most congested cases of $1500 \mathrm{veh} / \mathrm{h}$ and $2000 \mathrm{veh} / \mathrm{h}(+2 \%)$. In regards to fuel efficiency for the cars, the highest gain is seen at $1000 \mathrm{veh} / \mathrm{h}(+4 \%)$, as well as at the most congested cases of $1500 \mathrm{veh} / \mathrm{h}$ and $2000 \mathrm{veh} / \mathrm{h}$ (around $+2 \%$ ).

When the BSP scheme is implemented on a road network, it can be seen that cars emit more greenhouse gases, whereas buses are more environmentally friendly, as the amount of GHG they emit reduces (Figure 3, b, c, d). More specifically, cars emit more $\mathrm{HC}, \mathrm{CO}$ and $\mathrm{NOx}$ (7\% more $\mathrm{HC}$ and $\mathrm{CO}$ and 5.4\% more NOx) when BSP is applied to the current traffic flow (1500 veh/h). The lowest change in emissions for cars between BSP and mixed-traffic, and hence the most environmentally friendly, is observed at $1000 \mathrm{veh} / \mathrm{h}(+1.2 \%$ for $\mathrm{HC},+2.3 \%$ for $\mathrm{CO}$ and $+0.2 \%$

47 for NOx). The difference between the two schemes in terms of GHG emissions is less when 
1 buses are examined. However, buses emit the least amount of greenhouse gases when traffic 2 flow is at $1500 \mathrm{veh} / \mathrm{h}(-2.8 \%$ for $\mathrm{HC},-2.6 \%$ for $\mathrm{CO}$ and $-2.3 \%$ for NOx $)$. At the traffic flow that 3 cars are the most environmentally friendly (1000 veh/h), buses emit less HC (-1.6\%), less CO ($4 \quad 1.6 \%)$ and less NOx (-1.2\%) compared to the no BSP scheme.
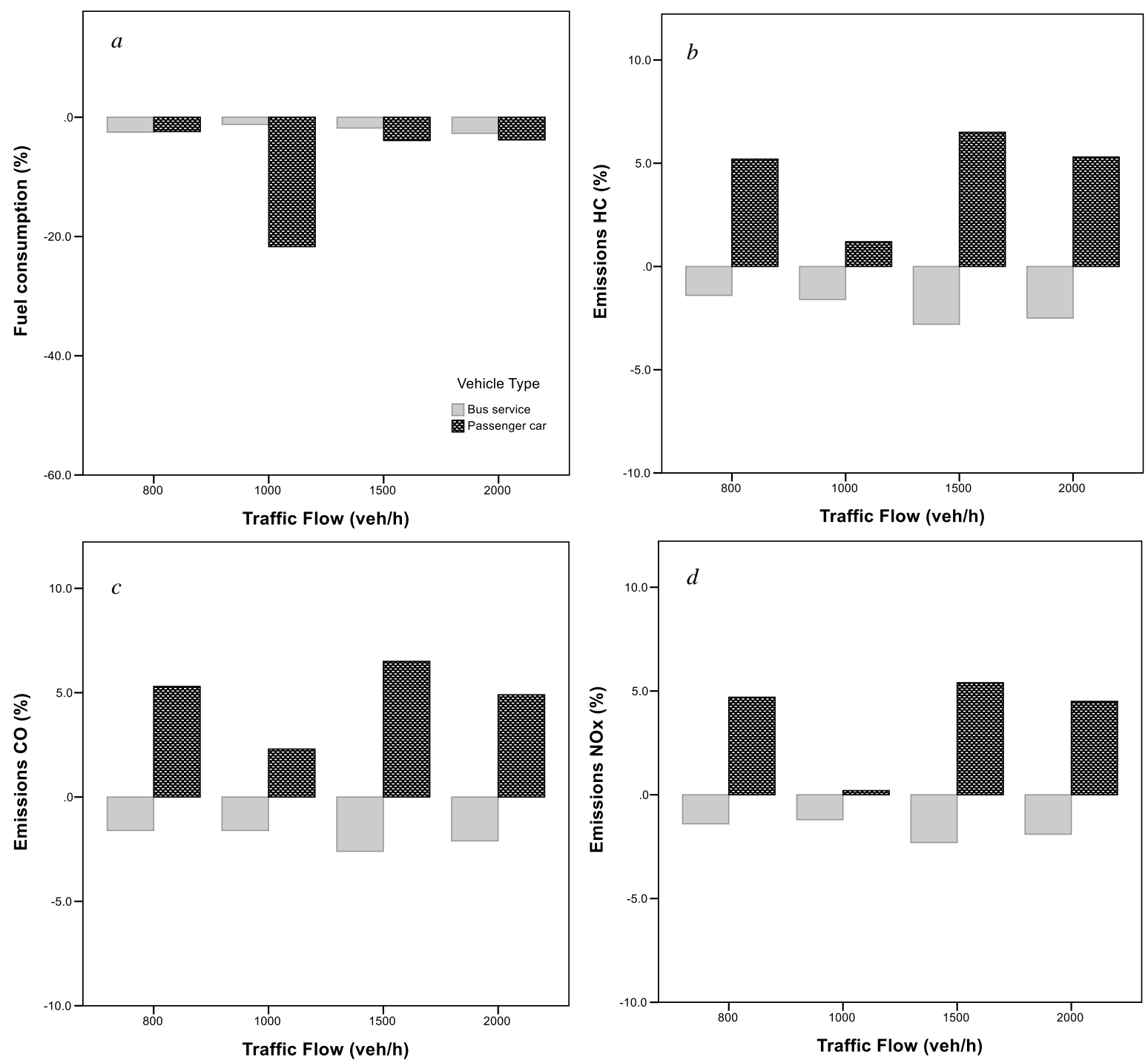

FIGURE 3 Percentage change of fuel consumption and emissions for cars and buses when BSP is applied.

\section{DISCUSSION AND CONCLUSIONS}

Microscopic traffic simulation has been used to examine the effect of two bus priority measures on the environment, and more specifically on reducing fuel consumption and GHG emissions. The tested bus priority measures included bus lanes and bus signal priority.

The selected road network presents high congestion, especially during peak hours, and is located in a district of Santiago, Chile that is served by a number of bus routes. Bus lanes are not included in the current road infrastructure; hence a mixed-traffic lane usage is experienced by road users. The selected bus route, $\mathrm{C} 01$, serves the 16 bus stops of the examined road network 
with 26 buses/h, whilst the current traffic flow is $1500 \mathrm{veh} / \mathrm{h}$. According to Vuchic (20), traffic flow should be less or equal to $1000 \mathrm{veh} / \mathrm{h}$ for the bus lane measure to be implemented. Therefore, the influence of traffic flows lower than the current, such as $1000 \mathrm{veh} / \mathrm{h}$ and 800 $\mathrm{veh} / \mathrm{h}$, was also investigated.

The results have shown that both bus priority measures contribute towards the improvement of the environment by reducing the amount of consumed energy and emitted GHG, especially by the bus service. In particular, for the bus lane scheme, limiting the traffic flow of this road network, or any other road network of similar characteristics, to $800 \mathrm{veh} / \mathrm{h}$ would result in the highest reduction of energy consumption and GHG emissions by both the cars and the bus service. However, the current traffic flow of the examined network is much higher than 800 $\mathrm{veh} / \mathrm{h}$, hence transport authorities will need to think of ways to reduce traffic on this street, as well as to direct road users towards more sustainable means of transport, such as public transport modes and cycling. This may not seem to be too hard to achieve, as, with the implementation of bus lanes, car users will experience lower average speeds which will then have an effect on their journeys by making them longer. As a result, car users will be dissatisfied by this experience and they might start searching for alternative means for their everyday commute, turning away from private cars. At the same time, bus services will be reaching higher average speeds and bus passengers will be reaching their destination much quicker than car users. Bus passenger satisfaction of the provided service will increase and passenger demand would be expected to follow the same trend.

The environmental impact of the BSP scheme is not as high as that of the bus lane scheme for cars and buses, but it also reduces energy consumption and emissions for the bus service. This reduction can be seen at all traffic flows for the bus service, nevertheless the BSP scheme has the biggest effect when applied at the current traffic flow $(1500 \mathrm{veh} / \mathrm{h})$. Therefore, if transport authorities decide to apply this measure without making any changes to the road infrastructure or finding ways to redirect traffic, the environmental improvement will be particularly apparent. When the results are considered in the case of cars, energy consumption and GHG emissions increase, however for a traffic flow of $1000 \mathrm{veh} / \mathrm{h}$ this increase is minimal.

At all traffic flows and when BSP is applied, road users of the main artery enjoy higher average speeds and hence shorter journeys. In the short run, this has the advantage of high satisfaction for both the car users and the bus passengers. However, considering the long run consequences, such a measure has the implication of not promoting active transportation. Car users will continue using their cars, as the applied bus priority scheme works to their advantage, and more and more people will be choosing the comfort of their private car compared to the provided public transport service or other more sustainable modes of transport. In order to achieve the highest possible environmental benefit, and accomplish the 2050 goals in regards to the global environmental challenges, transport authorities should be proposing solutions that turn road users away from their private cars. The additional benefits of such implementations would reflect on people's health and wellbeing and would dramatically reduce obesity (23).

Subsequently, it is evident that the 'Bus Only Lane' priority scheme has the highest and enduring environmental impact and contributes the most to the reduction of fuel consumption and emissions of cars and buses when applied at traffic flows up to $1000 \mathrm{veh} / \mathrm{h}$. To implement this measure, transport authorities as well as policy makers will need to enforce the creation of bus lanes dedicated solely to the bus service and to find ways to reduce the current traffic flow to at least $1000 \mathrm{veh} / \mathrm{h}$. Changing societal habits can be the biggest challenge, therefore it is essential that road monitoring is taking place to ensure that bus lanes are respected by car users. In terms of reducing the traffic flow, directing traffic to adjacent arteries will only shift the problem to 
other areas. The most effective way of achieving traffic flow reduction is by attracting people to public transport modes, and this should be taken on board by transport authorities. In the case that an immediate environmental improvement is required, transport authorities can implement the BSP scheme to the current traffic flow $(1500 \mathrm{veh} / \mathrm{h})$ and road infrastructure (mixed-traffic), but this measure does not ensure long-term reduction in fuel consumption and emissions.

Transport policy worldwide is encouraging people to use more active modes of transport in preference to the car, as it provides the means for non-car users to travel distances that are too great to walk without difficulty. Public transport, especially buses, plays an important part in the implementation of this policy. Organisations such as the Active Living Research programme and the Department of Transportation in the USA, and the WHO Regional Office in Europe provide information on how transport and mobility infrastructure have a positive impact on health (e.g. reduce obesity and improve respiratory diseases) and employability, reduce societal inequalities and increase connectivity (22). The critical role of urban planners and policy makers in this is greatly highlighted. The findings of this study show that priorities for buses, such as bus lanes and bus signal priority, have effects not only in reducing bus travel times, but also in fuel consumption and GHG emissions. These outcomes can be used in mass media campaigns to inform people of the benefits of active transportation, for the introduction of schemes aimed to reduce congestion - such as the congestion charge zone scheme in London, UK, for the reduction of street parking and the promotion of multi-modal transportation. The authors also believe that the results presented in this study can be taken as an example by other developing or rapidly growing countries with the aim to reduce their local energy consumption and GHG emissions challenges. Such countries can be other Latin American countries with similar road networks and China. It is undoubtable that advanced cities in the US and Europe can also benefit from these findings. Road networks in cities like Los Angeles, New York, Munich and many others, that present high congestion and mixed-traffic lane usage can reduce their energy consumption and emissions by following the recommendations of this paper.

Despite the significance of the outcomes of this study, the employed methods are subject to limitations and weaknesses that need to be recognised. TSIS-CORSIM is a software developed in the early 2000s, and therefore the values considered in the environmental tables and used to calculate fuel consumption and emission are outdated and reflect only a part of the vehicle fleet (mainly diesel cars) using the roads nowadays. Re-running the simulation model using updated environmental tables will increase the accuracy of the environmental impact of the proposed measures. Furthermore, considering a more diverse vehicle fleet - such as trucks, articulated and double decker buses - as well as the application to a road network, will provide a more complete overall picture of the environmental problems that need to be addressed.

As part of their future work, the authors consider testing the effect of bus acceleration on the environment. Buses are modelled in the examined network to accelerate and decelerate at 2.5 $\mathrm{m} / \mathrm{s}^{2}$ (absolute value). It has been shown by previous studies that the reduction of a vehicle's acceleration can greatly reduce fuel consumption and GHG emissions and increase passenger safety (24-27). A bus acceleration of $1.5 \mathrm{~m} / \mathrm{s}^{2}$ or lower has been proven to improve comfort during bus journeys and to enable passengers to walk naturally inside the bus when searching for a seat (28). This, however, cannot be achieved with the TSIS-CORSIM software as it is using a model to calculate fuel consumption and emissions which is not sensitive to changes in acceleration. An alternative approach will have to be followed.

\section{ACKNOWLEDGEMENTS}


1 This is a preliminary work done for the "City-Wide Analysis to Propel Cities towards Resource

2 Efficiency and Better Wellbeing" project, which is part of the Chinese Low Carbon Cities

3 Development and is funded by EPSRC (EP/N010779/1). The authors would like to thank Redbus

$4 \quad$ Urbano for their help in providing the necessary data for this study.

5

6 AUTHOR CONTRIBUTION STATEMENT

7 The authors confirm contribution to the paper as follows: study conception and design: Xenia

8 Karekla, Rodrigo Fernandez, Nick Tyler; data collection: Xenia Karekla, Rodrigo Fernandez;

9 analysis and interpretation of results: Xenia Karekla, Rodrigo Fernandez, Nick Tyler; draft

10 manuscript preparation: Xenia Karekla. All authors reviewed the results and approved the final

11 version of the manuscript. 


\section{REFERENCES}

2 1. WHO. Urban population growth [Internet]. WHO. 2017. Available from:

3 http://www.who.int/gho/urban_health/situation_trends/urban_population_growth_text/en/

42 Dena Levitz. How do greenhouse gas emissions compare in cities around the world? [Internet]. Hopes \& Fears. 2015. Available from: http://www.hopesandfears.com/hopes/city/city_index/216917-city-index-carbonemissions

3. Oxfam International. Extreme Carbon Inequality [Internet]. 2017. Available from: https://www.oxfam.org/en/research/extreme-carbon-inequality

4. WHO. Protecting health from climate change. [Internet]. 2008. Available from: http://www.who.int/world-health-day/toolkit/report_web.pdf

5. US Department of Transportation. Livability and Sustainability [Internet]. 2012. Available from: https://www.fhwa.dot.gov/publications/research/general/utc/2012webinar/sustainability/i ndex.cfm

6. International Energy Agency. Transport, Energy and CO2 [Internet]. 2009 p. 418. Available from: https://www.iea.org/publications/freepublications/publication/transport2009.pdf

7. European Environment Agency. Greenhouse gas emissions from transport [Internet]. European Environment Agency. Available from: https://www.eea.europa.eu/data-andmaps/indicators/transport-emissions-of-greenhouse-gases/transport-emissions-ofgreenhouse-gases-10

8. US EPA. Global Greenhouse Gas Emissions Data [Internet]. US EPA. 2016. Available from: https://www.epa.gov/ghgemissions/global-greenhouse-gas-emissions-data

9. ICCT. International Council on Clean Transportation - China [Internet]. 2017. Available from: http://www.theicct.org/china

10. European Commission. Reducing emissions from transport [Internet]. Climate Action European Commission. 2016. Available from: https://ec.europa.eu/clima/policies/transport_en

11. Raúl O’Ryan, Daniel Sperling, Mark Delucchi, Tom Turrentine. Transportation in Developing Countries: Greenhouse Gas Scenarios for Chile. [Internet]. Center for Climate and Energy Solutions; 2002. Available from: https://www.c2es.org/docUploads/transportation_chile.pdf

12. Button K. Transport Economics, 3rd Edition. 3rd Revised edition. Aldershot, Hants, England ; Northampton, MA: Edward Elgar Publishing Ltd; 2010. 528 p.

13. Creutzig F, Baiocchi G, Bierkandt R, Pichler P-P, Seto KC. Global typology of urban energy use and potentials for an urbanization mitigation wedge. Proc Natl Acad Sci. 2015 May 19;112(20):6283-8.

14. World Energy Council. Global Transport Scenarios 2050 [Internet]. 2011. Available from: https://www.worldenergy.org/wpcontent/uploads/2012/09/wec_transport_scenarios_2050.pdf

15. Cueto JL, Petrovici AM, Hernández R, Fernández F. Analysis of the Impact of Bus Signal Priority on Urban Noise. Acta Acust United Acust. 2017 Jul 1;103(4):561-73.

16. Li L, Persaud B, Shalaby A. Using micro-simulation to investigate the safety impacts of transit design alternatives at signalized intersections. Accid Anal Prev. 2017;100:123-32. 
17. Oguchi T, Mitsuyasu A, Oshima D, Imagawa T. An evaluation study on advanced public transport priority system using traffic simulation. Int J Urban Sci. 2017 Aug $1 ; 21(\sup 1): 43-53$.

18. Instituto Nacional de Estadistica. (Spanish Statistical Office). Transport of passengers statistic [Internet]. 2017. Available from: http://www.ine.es/en/welcome.shtml

19. Schirmer, W. N., Olanyk, L. Z., Guedes, C. L. B., Quessada, T. P., Ribeiro, C. B., \& Capanema, M. A. Effects of air/fuel ratio on gas emissions in a small spark-ignited nonroad engine operating with different gasoline/ethanol blends. Environmental Science and Pollution Research, 2017 24;25: 20354-20359.

20. Vuchic VR. Urban Transit Systems and Technology. 1 edition. Hoboken, N.J: John Wiley \& Sons; 2007. 624 p.

21. Fernandez R. A Study of Bus Operations on Arterial Roads by Simulation. ITE. 2003;73(4):77-81.

22. McGill R. Fuel Consumption and Emission Values For Traffic Models. Washington, DC: FHWA; 1985 May. Report No.: FHWA-RD-85-053.

23. ARUP, BRE, University College London, AREA Research. Health + Mobility: a design protocol for mobilising healthy living [Internet]. 2016 Oct [cited 2016 Oct 20]. Available from: http://publications.arup.com/publications/h/health_and_mobilityl

24. Pampel SM, Jamson SL, Hibberd DL, Barnard Y. How I reduce fuel consumption: An experimental study on mental models of eco-driving. Transp Res Part C Emerg Technol. 2015 Sep 1;58:669-80.

25. Lai W-T. The effects of eco-driving motivation, knowledge and reward intervention on fuel efficiency. Transp Res Part Transp Environ. 2015 Jan 1;34:155-60.

26. Martin NPD, Bishop JDK, Choudhary R, Boies AM. Can UK passenger vehicles be designed to meet 2020 emissions targets? A novel methodology to forecast fuel consumption with uncertainty analysis. Appl Energy. 2015 Nov 1;157:929-39.

27. Walnum HJ, Simonsen M. Does driving behavior matter? An analysis of fuel consumption data from heavy-duty trucks. Transp Res Part Transp Environ. 2015 May 1;36:107-20.

28. Karekla X. Improving Accessibility of Public Transport Systems: The Influence of Double-Decker Bus Acceleration on Passenger Movement [PhD]. University College London; 2016. 